\title{
OPTIMAL CONFIGURATIONS OF WAVE ENERGY CONVERTER ARRAYS WITH A FLOATING BODY
}

\author{
Wanchao Zhang', \\ Hengxu Liu', \\ Xuewei Zhang', \\ Liang Zhang ${ }^{1}$, \\ Muhammad Aqeel Ashraf ${ }^{2}$, \\ ${ }^{1}$ College of Shipbuilding Engineering, Harbin Engineering University, Harbin,China \\ ${ }^{2}$ Faculty of Science and Natural Resources, University Malaysia Sabah 88400 Kota Kinabalu Sabah, \\ Malaysia
}

\begin{abstract}
An array of floating point-absorbing wave energy converters (WECs) is usually employed for extracting efficiently ocean wave energy. For deep water environment, it is more feasible and convenient to connect the absorbers array with a floating body, such as a semi-submersible bottom-moored disk, whose function is to act as the virtual seabed. In the present work, an array of identical floating symmetrically distributed cylinders in a coaxial moored disk as a wave energy device is proposed The power take-off (PTO) system in the wave energy device is assumed to be composed of a linear/nonlinear damper activated by the buoys heaving motion. Hydrodynamic analysis of the examined floating system is implemented in frequency domain. Hydrodynamic interferences between the oscillating bodies are accounted for in the corresponding coupled equations. The array layouts under the constraint of the disk, incidence wave directions, separating distance between the absorbers and the PTO damping are considered to optimize this kind of WECs. Numerical results with regular waves are presented and discussed for the axisymmetric system utilizing heave mode with these interaction factors, in terms of a specific numbers of cylinders and expected power production.
\end{abstract}

Keywords: Wave energy; cylinder array; power take-off; hydrodynamic analysis

\section{INTRODUCTION}

The slow-speed periodic waves with large forces can cause periodic resonant motion of buoys relative to a reactant through which the wave energy can be extracted. This oscillating bodies utilizing heave mode which have small horizontal dimensions compared with the incident wave length have been defined as the simplest point-absorber wave energy converters. In the real sea, especially in deep water, the absorbers are often deployed in arrays connected to a bottom moored disk which makes them more stable, reliable and cost-effective. However, in such case, within the absorbers array, the interactions between the bodies due to diffracted waves and waves radiated by the oscillations of the bodies will occur. The wave energy conversion ability of the WECs array can be strongly impacted by these interactions depending on the incident waves, separating distances between the bodies and array layouts, as well as the PTO damping.

The pioneer work of studying the interactions that affect the WECs array can be traced to Budal [1] who put forward an interaction factor to assess the effect of the interaction depended on the array configurations and the separating distances between the bodies. For his study, the absorbers are assumed to be small to neglect the scattered wave. Subsequent work are carried out by Falnes and Budal [2] who affirmed that whether the effect is constructive depends on the incident wave frequencies and the array layouts. Based upon their theories, McIver [3] analyzed the wave power absorption performance of the array by setting unequal spacing of devices and constraining the amplitudes of device motions 
through surge or heave motions. He found that the wave power absorption performance of the array is dramatically depended on the incidence wave directions in heave and less sensitivity to that in surge. Fitzgerald and Thomas [4] proceeded further in this direction, studying the effect of the variations of array geometries and incident wave directions on the wave power conversion performance of a five hemishperes array. The interaction factor is found to be equal to unity over all the incident wave directions $0 \sim 2 \pi$. The most recent studies on the interactions correspond to researchers [5-12] Weller et al. [6] examined the interaction factors of the floaters array through experiment measurement in regular and irregular waves and found that whether the interaction is positive depends on the displacements of the floaters, incident wave periods and the performance of adjacent devices. Child and Venugopal [7] optimized a generic point absorbers array by adjusting the incident wave directions and the array layouts through two different methods (parabolic intersection and genetic algorithm methods). Babarit [8] examined two twobody oscillating WECs with heave or surge motions and demonstrated that when the separating distance is over $2000 \mathrm{~m}$, the interactions are wake and can be negligible which is very important in the preliminary design of a WECs farm in the real sea. Most of the latest studies carried out on the WECs array and power optimization correspond to [13-17]. Andres et al. [14] exceed further in this kind of twobody oscillating WEC through varying the array layout, the separating distance, the number of WECs and the wave directions. Their results suggested that the wave directions and number of WECs are major factors to make the interactions constructive and the triangular array layout may be more adaptable in irregular waves. Finally, Goteman et al. [16] made it more extreme on the study of the number of WECs. They optimized a wave energy park which contains over 2000 point absorbers including global geometries, number of devices, separating distance between devices and sea states by using an approximate analytical method.

Most of the WEC devices considered in the former studies are isolated and installed directly connected to the sea floor which makes them possible with typical large separating distances of few hundreds of meters. However, for practical reasons (limitation of sea area and economic cost for example), the arrays of WECs cannot be isolated and sparse especially in such water depth. It may be more credible and adaptable to connect the absorbers array with a floating moored disk on which the PTO mechanism can be installed. Therefore it is particularly significant and necessary to analyze the interaction factors under the constraint of the disk such as the separating distances, array layouts and PTO damping. As a matter of fact, the PTO damping also has a great effect on the wave energy conversion performance even though few of researchers have paid their attention to the PTO damper (linear or nonlinear). The special work can correspond to Sheng et al. [19], Sheng and Anthony [20,21] who optimized the PTO damper considering the variation of PTO damping coefficients and velocity index.

The aim of this paper is to investigate the potential for wave energy extraction with an array of WECs at intermediate water depth considering different incident wave directions, array layouts, separating distances and PTO dampers. As the absorbers should work against the reactant to activate the actuator which is difficult to be installed on the seabed in such water depth, a moored reactant is needed. Thus, in this work, arrays of identical vertical floating absorbers symmetrically distributed over and sliding along some guides attached to a coaxial moored semi-submersible disk were proposed as a WEC device for extracting wave energy as shown in Fig.1. Numerical results concerning for the hydrodynamic coefficients and the wave excitation forces in heave are presented for a specific case of four identical cylinders over a plate considering different incident wave angles. Three special array layouts with varying wave directions, separating distances and linear PTO damper are conducted to examine the effect of these factors on the wave power absorption performance. Emphasis is given on the optimization of captured wave energy by choosing the appropriate PTO damping coefficients and velocity indexes.

\section{MATHEMATICAL MODEL}

The array of WECs employed in this paper is illustrated in Fig. 1 which contains a semi-submersible disk and $\mathrm{N}(\mathrm{N}=1$, $2, \ldots)$ floating absorbers. The multi-body oscillating system with mooring system is thought to be in static equilibrium in still water, and their vertical oscillations are measured relative to their positions of static equilibrium in this paper. Consistent with a frequency-domain treatment, sinusoidal incident waves at frequency $\omega$ are assumed. The linear superposition of diffraction and radiation forces follows from the small-amplitude assumption. Straightforward application of Newton's laws leads to the equations of motion for system in Fig.1.

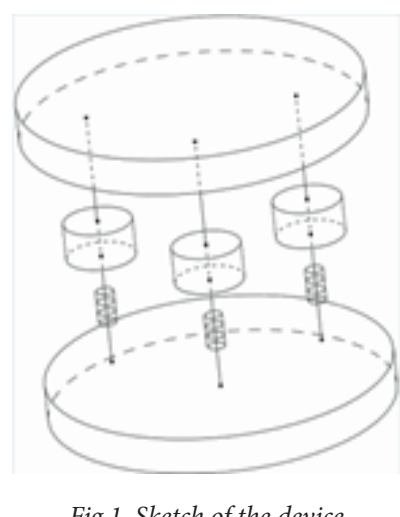

Fig.1. Sketch of the device

We denote the masses of the cylinders and the disk as mq $(q=0,1, \ldots, N)$. Here and hereafter, the subscript $q(q=0,1$, $2, \ldots, N)$ denotes body $q$ of this complex system. It represents the semi-submersible disk when $\mathrm{q}=0$ and the $\mathrm{q}$-cylinder when $\mathrm{q}=1,2, \ldots, \mathrm{N}$. In such case, the hydrostatic restoring force stiffness of body q can be denoted as $\mathrm{k}_{\mathrm{q}}=\rho g \mathrm{~S}_{\mathrm{q}}$. Here, $\rho$ denotes the density of sea water, $g$ denotes the acceleration due to gravity and $\mathrm{S}_{\mathrm{q}}$ denotes the water plane area of body $\mathrm{q}$. In this paper, the bottom structure of the disk is through to be 
a submerged plate. The support columns where the cylinders are installed are assumed to be small-scale components and have little effect on the hydrodynamic performance. In such case, the water plane areas of the disk are assumed to be zero. Further, we define the controllable resistive load coefficient as $\mathrm{c}_{\mathrm{q}}$ which comprise the q-actuator activated by the cylinder $\mathrm{q}(\mathrm{q}=1, \ldots, \mathrm{N})$ and the disk $(\mathrm{q}=0)$. The equation of heave motion in regular waves for this system is

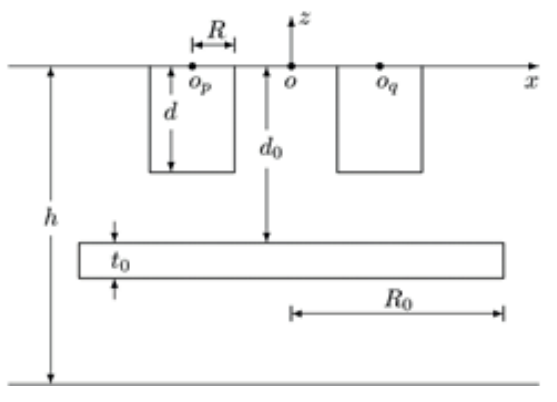

Fig.2. Simplified model and geometries

$$
\mathbf{M Z}=\mathbf{F}_{e x}+\mathbf{F}_{r}+\mathbf{F}_{s}+\mathbf{F}_{t}+\mathbf{F}_{P T O}
$$

with: $\mathbf{Z}=\mathfrak{R}\left(\bar{Z} e^{-i \omega t}\right)$ and $\dot{Z}, \ddot{Z}$ being, respectively, the velocity and acceleration vectors of the system in heave (1)

$\mathbf{M}=\operatorname{diag}\left(m_{0}, m_{1}, \cdots, m_{N}\right)$, the inherent mass matrix of the system

$\mathbf{F}_{e x}=\mathfrak{R}\left(\bar{F} e^{-i \omega t}\right)$, the excitation force vector per unit of wave amplitude, associated to the action of incident and diffracted wave fields upon the WECs

$\mathbf{F}_{r}=-\mathbf{M}_{\omega} \ddot{\mathbf{Z}}-\mathbf{B}_{\omega} \dot{\mathbf{Z}}$, the radiation force vector due to the bodies' oscillation in the otherwise calm water, in which $\mathrm{M}_{\omega}(\mathrm{pg})=\mu_{\mathrm{pg}}=\mu_{\mathrm{qp}}$ and $\mathrm{B}_{\omega}(\mathrm{p}, \mathrm{g})=\lambda_{\mathrm{pg}}=\lambda_{\mathrm{qp}}$ Here, $\mu_{\mathrm{pq}}$ and $\lambda \mathrm{pq}(\mathrm{p}$, $\mathrm{q}=0,1, \ldots, \mathrm{N})$ denote the added mass and damping coefficients of body $\mathrm{p}$ due to the oscillation of body $\mathrm{q}$.

$\mathrm{F}_{\mathrm{S}}=-\mathrm{K}_{\mathrm{s}} \mathrm{Z}$, the hydrostatic force vector of the system with $\mathrm{K}_{\mathrm{S}}=\operatorname{diag}\left(\mathrm{k}_{0}, \mathrm{k}_{1}, \ldots, \mathrm{k}_{\mathrm{N}}\right)$.

$\mathrm{F}_{\mathrm{t}}=-\mathrm{K} \mathrm{Z}$, the force vector of possible moorings. In this study, it was neglected, i.e. $\mathrm{K}_{\mathrm{t}}=0$.

$\mathrm{F}_{\text {РтO }}=-\mathrm{B}_{\text {РтO }} \mathrm{Z}$, the force vector of the actuators activated by the absorbers' and the disk's oscillations. $\mathrm{B}_{\text {Рто }}$ is the PTO damping coefficients matrix and $\mathrm{B}_{\text {Рто }}(\mathrm{q}, \mathrm{q})=\mathrm{c}_{\mathrm{q}-1}$, $\mathrm{B}_{\text {PTO }}(1, \mathrm{q})=\mathrm{B}_{\text {РTO }}(\mathrm{q}, 1)=-\mathrm{c}_{\mathrm{q}-1}$ with $\mathrm{q}=2, \ldots, \mathrm{N}+1$ and $\mathbf{B}_{P T O}(1,1)=\sum_{q=1}^{N} c_{q}$ with $c_{q}=C_{P T O}\left|\omega \bar{Z}_{0}-\omega \bar{Z}_{q}\right|^{\alpha}$. The superscript $\alpha$ is the velocity index. In this study, both the linear $(\alpha=0)$ and nonlinear $(\alpha>0)$ PTO damping forces are considered. The not listed elements in this matrix are zero.

In such case, in regular waves, the time-averaged wave power extracted by each absorber in the array per unit of wave amplitude can be calculated and expressed as

$$
\bar{P}_{q}=1 / 2 \omega^{2} c_{q}\left(\bar{Z}_{q}-\bar{Z}_{0}\right)\left(\bar{Z}_{q}-\bar{Z}_{0}\right)^{*}
$$

with $\mathrm{q}=1, \ldots, \mathrm{N}$ and the asterisk denotes the conjugate complex. For the whole array, the mean absorbed power is

$$
\bar{P}=\sum_{q=1}^{N} \bar{P}_{q}
$$

To obtain the excitation forces, added mass and radiation damping coefficients in heave, for linear water wave theory, it is convenient to decompose the velocity potentials in the fluid around the device as

$$
\Phi=\Phi_{D}+i \omega \sum_{p=1}^{N} A_{p} \Phi_{p} \quad \text { with } \quad \Phi_{D}=\Phi_{I}+\Phi_{S}
$$

Where $\Phi_{D}$ is the diffracted velocity potential which contains incident wave velocity potential $\Phi_{\mathrm{I}}$ and scattered wave velocity potential $\Phi_{S} . \Phi_{\mathrm{p}}$ stands for radiated velocity potential in heave of body $\mathrm{p}(\mathrm{p}=0,1, \ldots, \mathrm{N})$. According to the potential flow theory, the velocity potentials should satisfy:

Laplace's equation:

$$
\nabla^{2} \Phi_{D, p}=0 \quad \text { (in fluid) }
$$

Free surface condition:

$$
-f^{2} \Phi_{D, p}+g \partial_{z} \Phi_{D, p}=0 \quad(z=0)
$$

Bottom condition:

$$
\partial_{z} \Phi_{D, p}=0 \quad(z=-h)
$$

Radiation condition:

$$
\lim _{r \rightarrow \infty} \sqrt{r}\left(\partial_{r} \Phi_{D, p}-i k \Phi_{D, p}\right)=0
$$

Hull boundary condition on $\mathrm{S}^{(\mathrm{p})}$ :

$$
\partial_{n^{p}} \Phi_{q}=\delta_{p q} n_{3}^{q}, \partial_{n^{p}} \Phi_{D}=0
$$

Here the subscripts and superscripts $p, q(p, q=0,1, \ldots, N)$ denote the body $\mathrm{p}$ and body $\mathrm{q}, \mathrm{f}^{2}=\omega^{2} / \mathrm{g}$ with $\omega$ as the wave frequency and $g$ the acceleration due to gravity. The wave number $\mathrm{k}$ is defined by the dispersion equation ktanhkh=f $\mathrm{f}^{2}$ which is obtained by satisfying the boundary conditions. Here and hereafter, $\partial \mathrm{n}($.$) denotes the derivative in the direction of$ the outward unit normal vector $n$ to the mean wetted surface $\mathrm{S}^{(\mathrm{p})}$ of the body $\mathrm{p}, \delta_{\mathrm{pq}}$ is the Kornecker's symbol and $\mathrm{n}_{3}^{\mathrm{q}}$ is the normal component in the vertical direction.

Thus the non-dimensional first order force in heave direction is given by integrating the pressure on the hull and expressed by

$$
F_{p}=-i \omega / g R_{p}^{2} \int_{S^{(p)}} \Phi_{D} n_{3}^{p} d s \quad(p=0,1, \cdots, N)
$$

The added mass and the damping coefficients of body $\mathrm{p}$ by body q's oscillation can be defined by

$$
\mu_{p q}+i \lambda_{p q} / \omega=1 / R_{p}^{3} \int_{S^{(p)}} \Phi_{q} n_{3}^{p} d s \quad(p, q=0,1, \cdots, N)
$$

\section{ADDED MASS, RADIATION DAMPING AND WAVE FORCING}

As mentioned before, this paper is focus on accessing the factors that influence the wave energy conversion performance and optimizing the WECs array. The water depth and the geometries of the structures are not included in these influence factors though they may have a great influence on the calculated results. For simplicity of hydrodynamic 
calculation, the upper structures of the disk are assumed to be out of water and the bottom ones are simplified as a submerged plate in this wave power capturing process. In such case, the water depth and the geometries of the structures as shown in Fig. 2 should be confirmed and given as $\mathrm{h}=50 \mathrm{~m}$, $\mathrm{R} / \mathrm{h}=0.04, \mathrm{R}_{0} / \mathrm{R}=4.0, \mathrm{~d} / \mathrm{R}=2.5, \mathrm{~d}_{0} / \mathrm{R}=4.0$ and $\mathrm{t}_{0} / \mathrm{R}=0.5$ in this study.

A four cylinder array over a plate is considered in this paper and the array layouts are shown in Fig.3. Layout (a) is a square configuration consisting of two lines of absorbers. Layout (b) is a rhombus configuration consisting of two equilateral triangles. Finally, layout (c) is an equilateral triangle configuration with another absorber at the central point. Above all the three array layouts, the geometric center of the configurations and the center of the plate are coincident.

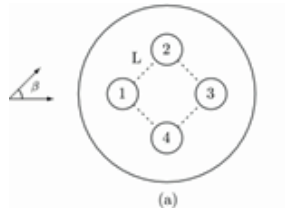

Fig.3. Layouts of the absorbers array
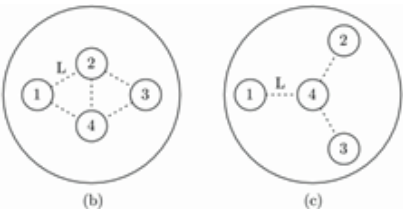

(c)
To provide the hydrodynamic coefficients $M_{\omega}, B_{\omega}$ and $\mathrm{F}_{\omega}$ for the optimization, a BEM based FORTRAN code is used. The calculated hydrodynamic coefficients are plotted in Fig. 4 in the case of array layout (a) with incident wave direction $\beta=0^{\circ}, 45^{\circ}$ and separating distance $L / R=0.5,1.5$. The illustrated added mass gives an expected result that the cross coupled added mass decreases with the increase of the separating distance. However, the added mass caused by the cylinder's own oscillation also shows the same trend. Particular attention should be given that the frequencydependent amplitude reduction of the added mass seems to be the same except those happen in the range of $[0.7,2.5]$ $\mathrm{rad} / \mathrm{s}$. In this frequency range, we notice a great change in the amplitude reduction with the variation of the separating distances. In the upper-right subgraph in Fig.4, the radiation damping stays the same outside this frequency range, and shows great change in the frequency range especially when the frequency $\omega=1.8 \mathrm{rad} / \mathrm{s}$ with different separating distances.
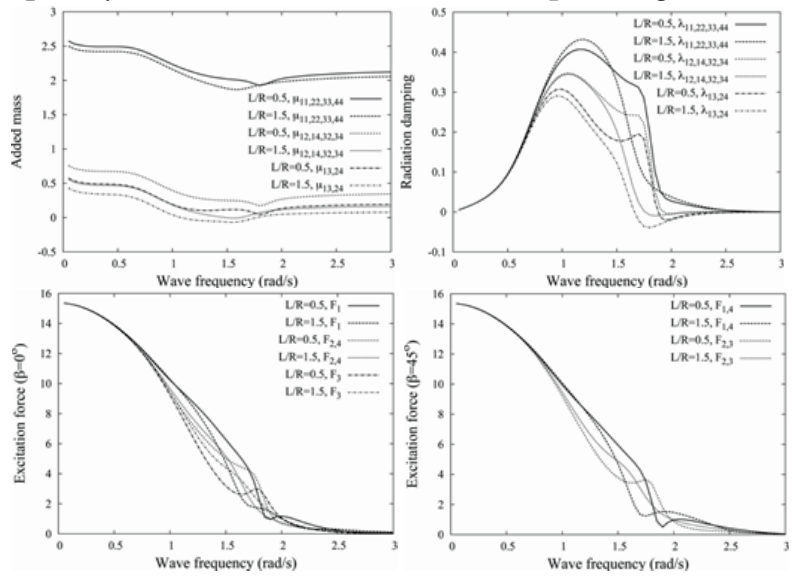

Fig.4. Non-dimensional added mass (upper-left), radiation damping (upperright) and wave excitation force (below) in array layout (a) with incident wave direction $\left(\beta=0^{\circ}, 45^{\circ}\right)$ and separating distance $(L / R=0.5,1.5)$
About the excitation forces, the similar phenomenon also occurs. The incidence angles and separating distances play major roles in determining the degree of hydrodynamic interference in the same frequency range. On these evidences, the effect of incident wave direction and separating distance are not significant outside this frequency range and therefore might reasonably be neglected when estimating damping and excitation forces which are highly correlated to the wave energy conversion.
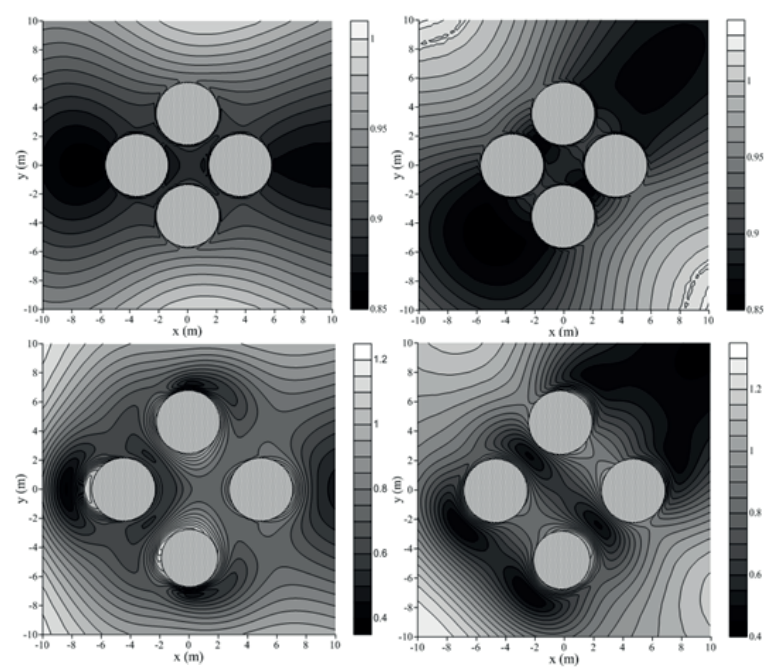

Fig.5. Non-dimensional elevations at the free surface in array layout (a) with incident wave direction $\left(\beta=0^{\circ}, 45^{\circ}\right)$ and separating distance $(L / R=0.5,1.5)$

\section{NUMERICAL RESULTS}

This section will deal with the factors that affect the wave energy conversion performance and optimizing this kind of WECs array. As mentioned before, the factors include the array layout, the separating distance, the incidence wave direction and the PTO damping. Of all the four factors, the PTO damping's effect includes the effect of the PTO damping type (linear and nonlinear) and that of the damping coefficient value. In such case, in the study of the first three factors, the PTO damping is assumed to be linear and the coefficient is set to be $10 \mathrm{kN} \cdot \mathrm{s} / \mathrm{m}$.

As is known to all, the wave energy conversion performances of oscillating-typed WECs are usually highly dependent on the incident wave frequency and natural frequencies of the oscillators in the analysis based upon the potential flow theory. In addition, the resonance condition is that the natural frequency of the absorber approaches the frequency of the incident wave. Thus, the undamped natural frequency $\omega_{n}$ of the absorber should be an important reference condition for the following analysis and defined as $\omega_{n}^{2}=k /(m+\mu)$. Here, $k$, $\mathrm{m}$ and $\mu$ denote the hydrostatic restoring force stiffness, the inherent mass and added mass of the absorber respectively. In such case, the range of the natural frequencies of the absorbers for the considered array layouts with different separating distances can be obtained and recorded in table 1 .

As shown in table 1, the natural frequency ranges are almost the same and in such case, the frequency being choose to analyze the factors will be near this frequency range [1.2, 
1.3]. Fig.5 7 show the wave energy conversion performance with separating distance for the considered three array layouts at frequency $\omega=1.0,1.25$ and $1.5 \mathrm{rad} / \mathrm{s}$ with different incident wave directions.

Table 1 Range of the natural frequencies

\begin{tabular}{ccccc}
\hline \hline Layout No. & $L / R=0.5$ & $L / R=1.0$ & $L / R=1.5$ & $L / R=2.0$ \\
\hline (a) & {$[1.216,1.255]$} & {$[1.218,1.258]$} & {$[1.220,1.259]$} & {$[1.222,1.260]$} \\
(b) & {$[1.217,1.251]$} & {$[1.220,1.256]$} & {$[1.223,1.258]$} & {$[1.225,1.260]$} \\
(c) & {$[1.220,1.256]$} & {$[1.223,1.258]$} & {$[1.226,1.260]$} & {$[1.229,1.261]$} \\
\hline \hline
\end{tabular}

\section{SEPARATING DISTANCE}

Considering the area restriction of the disk, the separating distance $\mathrm{L} / \mathrm{R}$ considered in this study is set in the range of $[0.5,2.0]$. Analyzing the following Fig. $6 \sim 8$, it is noticeable that the separating distance shows amazing effect on the wave conversion performance of this kind of WEC. For the considered array layouts, the effect of the distance are highly depend on the incident wave frequency. All the curves show more or less a similar pattern for the considered wave frequencies respectively. As shown in the left subgraphs in Fig. $6 \sim 8$, the captured power decrease first and then increase with the increase of the separating distance at the frequency $\omega=1.0 \mathrm{rad} / \mathrm{s}$ though it is not so obvious in the rhombus array layout. While at the frequency $\omega=1.25 \mathrm{rad} / \mathrm{s}$ as shown in the middle subgraphs, the captured power keeps increasing with the increase of the distance. The right subgraphs give an opposite trend with the left ones: increase first and then decrease.
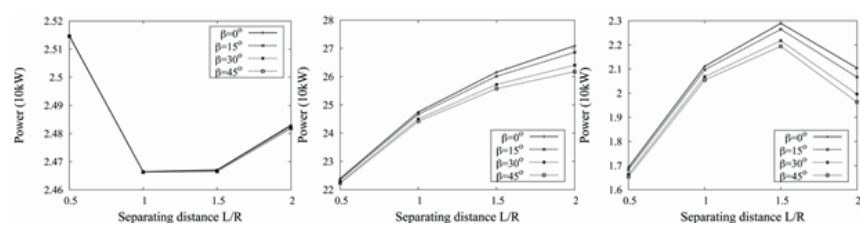

Fig.6. Captured wave power for array layout (a) with separating distance at different wave frequencies; Left: $\omega=1.0 \mathrm{rad} / \mathrm{s}$; Middle: $\omega=1.25 \mathrm{rad} / \mathrm{s}$; Right: $\omega=1.5 \mathrm{rad} / \mathrm{s}$
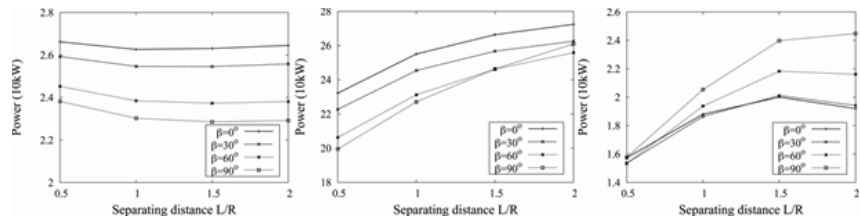

Fig.7 Captured wave power for array layout (b) with separating distance at different wave frequencies; Left: $\omega=1.0 \mathrm{rad} / \mathrm{s}$; Middle: $\omega=1.25 \mathrm{rad} / \mathrm{s}$; Right: $\omega=1.5 \mathrm{rad} / \mathrm{s}$

\section{ARRAY LAYOUT}

It can be observed from Fig6 8 that the behaviors of the WECs related to the array layouts are highly depended on the incident waves and the separated distances. As shown in the left subgraphs, the rhombus layout shows better conversion performance than the other two layouts just considering the maximal value though the square layout shows better stability. While at the wave frequency $\omega=1.25 \mathrm{rad} / \mathrm{s}$ as illustrated in the middle subgraphs, at a smaller separating distance such as $\mathrm{L} / \mathrm{R}=0.5$, the triangular array layout shows better conversion ability. However, with the increasing distance, the square layout shows better performance not only in the value of captured power but also in the stability with incidence wave direction. The right subgraphs depict the captured wave power at wave frequency $\omega=1.5 \mathrm{rad} / \mathrm{s}$ with different separated distances. It is noticeable that when the distance is small, the triangular array layout shows better performance, however, with the increasing distance, the rhombus one is better especially when the incident angle is $90^{\circ}$. Particular attention should be given to that the square layout shows excellent performance not only in the value of captured power but also in the stability with incidence wave direction.
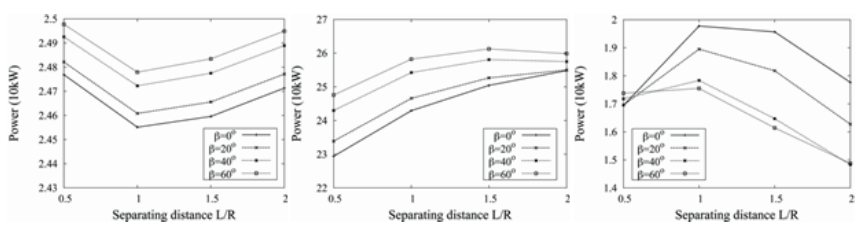

Fig.8 Captured wave power for array layout (c) with separating distance at different wave frequencies; Left: $\omega=1.0 \mathrm{rad} / \mathrm{s}$; Middle: $\omega=1.25 \mathrm{rad} / \mathrm{s}$; Right: $\omega=1.5 \mathrm{rad} / \mathrm{s}$.

\subsection{INCIDENCE WAVE DIRECTION}

The effects of the incident wave angles are so obvious in all the subgraphs and depend on the layouts. In such case, the analysis should be performed taking into account each layout. For the square array layout, at a lower wave frequency, the angle shows little effect, while at higher wave frequencies, the captured wave power decreases with the increasing incident angle in the considered entire separated length range. For the rhombus layout, at the frequencies $\omega=1.0$ and $1.25 \mathrm{rad} / \mathrm{s}$, the captured wave power decreases with the increasing incident angle. The difference, however, is that when the separating distance $L / R$ is larger than 1.5 , the incident angle $\beta=90^{\circ}$ leads to more excellent performance than $\beta=60^{\circ}$. While at higher frequency $\omega=1.5$, the captured wave power increases with the increasing incident angle. There is something special that the incident angle has little effect on the conversion ability when the angles are small. For the triquetrous layout, a larger incident angle leads to more excellent conversion performance when the wave frequencies $\omega=1.0$ and $1.25 \mathrm{rad} / \mathrm{s}$, while at the frequency $\omega=1.5 \mathrm{rad} / \mathrm{s}$, the smaller incident angle shows better performance.

\section{PTO DAMPING}

As mentioned before, the influence of the PTO damping contains the influence of the PTO damping coefficient $\mathrm{C}_{\text {Рто }}$ and that of the damping type embodied in the velocity index. In such case, we analyze their influence from these two aspects 
in the case of array layout (a) with separating distance L/ $\mathrm{R}=2.0$ and incident wave angle $\beta=0^{\circ}$.

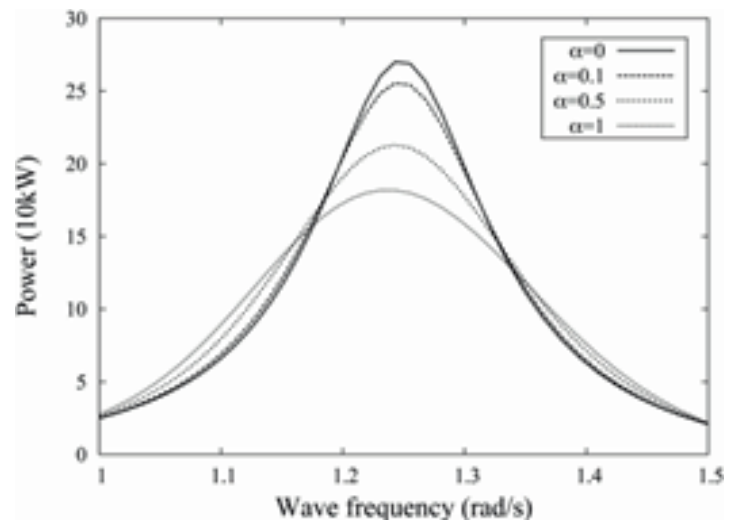

Fig.9. Captured wave power with wave frequencies when $C_{\text {PTO }}=10 \mathrm{kN} \cdot \mathrm{s} / \mathrm{m}$

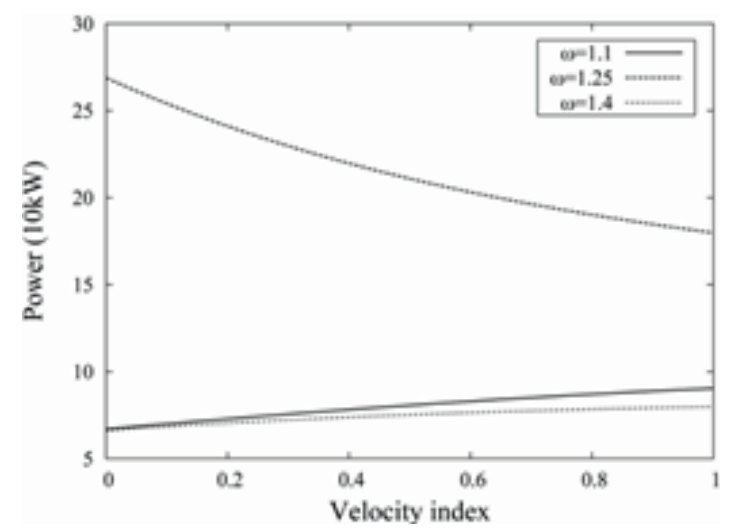

Fig.10. Captured wave power with velocity indexes when $C_{P T O}=10 \mathrm{kN} \cdot \mathrm{s} / \mathrm{m}$

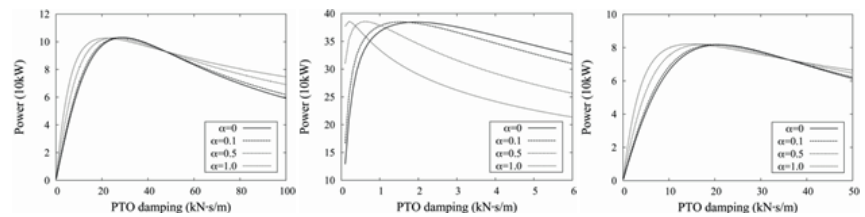

Fig.11 Captured wave power with PTO damping coefficients in case of layout (a); Left: $\omega=1.1 \mathrm{rad} / \mathrm{s}$; Middle: $\omega=1.25 \mathrm{rad} / \mathrm{s}$; Right: $\omega=1.4 \mathrm{rad} / \mathrm{s}$

- Velocity index: Fig.9 depicts the captured wave power of square array layout with different velocity indexes when the PTO damping coefficient $\mathrm{C}_{\text {РтO }}=10 \mathrm{kN} \cdot \mathrm{s} / \mathrm{m}$. It is observed that the velocity index has a great effect on the wave energy conversion performance. In the frequency ranges [1.0, 1.17] $\mathrm{rad} / \mathrm{s}$ and $[1.33,1.5] \mathrm{rad} / \mathrm{s}$, the higher index shows better performance, such as $\alpha=1$ (quadratic). However, in the range of $[1.17,1.33] \mathrm{rad} / \mathrm{s}$, the lower velocity index shows better performance, such as $\alpha=1.0$ (quadratic PTO). Special attention should be given to the frequency where the peak value of the power occurs. There is a slight left shift in the peak frequency with the increasing velocity index. The Fig.9 shows the effect of velocity index on the captured power with considered three frequencies which further affirms the previous conclusions.

- PTO damping coefficient: The effect of the coefficient value can be seen from Fig.11. It is noticeable that the wave energy conversion abilities are highly depended on the PTO damping coefficients and the incident wave frequencies. The captured power increases first and then decreases with the increasing PTO damping coefficients. The damping coefficient where the peak value of the power occurs decreases with the increasing velocity index though the peak values are the same. However, at lower frequencies (such as $\omega=1.1 \mathrm{rad} / \mathrm{s}$ ) and higher frequencies (such as $\omega=1.4 \mathrm{rad} / \mathrm{s}$ ), the curves of corresponding higher velocity indexes are seen to be more smooth and the corresponding captured powers stay relative high in a larger range of the damping coefficients. At the frequencies around the natural frequency of the absorber such as $\omega=1.25 \mathrm{rad} / \mathrm{s}$, the conversion performance seems to be more excellent as the values of the captured power are relative high in the considered range of PTO damping coefficients.

\section{DISCUSSION AND CONCLUSION}

A four absorbers array connected to a semisubmersible disk is composed as a wave energy converter to extract ocean wave energy in this study. The interactions of the hydrodynamics and PTO mechanisms between the bodies are accounted for in the proposed corresponding coupled equations in frequency domain. A BEM based FORTRAN code is programmed to calculate the hydrodynamics of the oscillating system. The separating distance, array layout, incident wave direction and the PTO damping are considered for the optimization of the proposed WEC. The calculation results show that:

(1) The hydrodynamic characteristics of the disk combined WEC show good stability at very low and high frequencies. In particular, the frequency-dependent amplitude of the added mass decreases with the increasing separating distance and the reduction seems to be the same at these frequencies. However, in the frequency range $[0.7,2.5] \mathrm{rad} / \mathrm{s}$, they are highly affected by the separating distances and the incident wave directions for each considered array layout.

(2) The effects of the separating distance on the wave energy conversion performance are highly depended on the incident wave frequency. The captured wave powers decrease first and then increase with the increasing separating distance at the frequency $\omega=1.0 \mathrm{rad} / \mathrm{s}$ and shows the opposite trend when $\omega=1.5 \mathrm{rad} / \mathrm{s}$. While at the frequency $\omega=1.25 \mathrm{rad} / \mathrm{s}$, the captured power keeps increasing with the increase of the distance. Thus, the separating distance should be determined by the frequencies in its operation sea area.

(3) The square array layout shows better performance than the rhombus and triangular ones not only in the value of captured power but also in the stability with incidence wave direction. However, at the frequencies $\omega=1.25$ and $1.5 \mathrm{rad} / \mathrm{s}$, the triangular one shows better performance when the separating distances are small. In summary, the square array layout is more appropriate considering the restriction of the disk.

(4) The incidence wave direction affects the conversion ability depending on the choice of the array layout and the wave frequency. The square one is not sensitive to changes in the incident wave angle. The rhombus and triangular ones show the opposite trend. 
(5) The influence of the PTO damping is reflected in two aspects, the damping type (velocity index) and the damping coefficient. The higher velocity index shows better applicability in the real random sea area. For different velocity indexes, the captured power can reach the maximum value by changing the damping coefficient.

\section{ACKNOWLEDGEMENT}

This paper is financially supported by the National Natural Science Foundation of China (No.51106034, No.51579055 and No.51509048).

\section{REFERENCE}

1. Budal K (1977). Theory for absorption of wave power by a system of interacting bodies. Journal of Ship Research, 21:248-253.

2. Falnes J, Budal K (1982). Wave-power absorption by parallel rows of interacting oscillating bodies. Applied Ocean Research, 4:194-207.

3. McIver P (1994). Some hydrodynamic aspects of arrays of wave energy devices. Applied Ocean Research, 19:283-291.

4. Fitzgerald C, Thomas G (2007). A preliminary study on the optimal formation of an array of wave power devices. In: Proceedings of the 7th European Wave and Tidal Energy Conference, Porto, Portugal.

5. Garnaud X, Mei CC (2010). Comparison of wave power extraction by a compact array of small buoys and by a large buoy. IET Renewable Power Generation.4 (6):519-530.

6. Weller SD, Stallard TJ, Stansby PK (2010). Experimental measurements of irregular wave interaction factors in closely spaced arrays. IET Renewable Power Generation.4 (6):628-637.

7. Child BFM, Venugopal V (2010). Optimal configurations of wave energy device arrays. Ocean Engineering, 37:1402-1417.

8. Babarit A (2010). Impact of long separating distances on the energy production of two interacting wave energy converters. Ocean Engineering, 37:718-729.

9. Borgarino B, Babarit A, Ferrant P (2012). Impact of wave interactions effects on energy absorption in large arrays of wave energy converters. Ocean Engineering, 41:79-88.

10. Wolgamot HA, Taylor PH, Taylor RE (2012). The interaction factor and directionality in wave energy arrays. Ocean Engineering, 47:65-73.
11. Heikkinen H, Lampinen MJ, Boling J (2013), Analytical study of the interaction between waves and cylindrical wave energy converters oscillating in two modes. Renewable Energy, 50:150-160.

12. Sharkey F, Bannon E, Conlon M, Gaughan K (2013), Maximising value of electrical networks for wave energy converter arrays. International Journal of Marine Energy, 1:55-69.

13. Goteman M, Engstrom J, Eriksson M, Isberg J, Leijon M (2014). Analytical and numerical approaches to optimizing fluid-structure interactions in wave energy parks. Iwwwfb. naoe.eng.osaka.

14. Andres AD, Guanche R, Meneses L, Vidal C, Losada IJ (2014). Factors that influence array layout on wave energy farms. Ocean Engineering, 82:32-41.

15. Goteman M, Engstrom J, Eriksson M, Isberg J, Leijon M (2014). Methods of reducing power fluctuations in wave energy parks. Journal of Renewable and Sustainable Energy, 6: 043103.

16. Goteman M, Engstrom J, Eriksson M, Isberg J (2015). Optimizing wave energy parks with over 1000 interacting point-absorbers using an approximate analytical method. International Journal of Marine Energy, 10:113-126.

17. Konispoliatis DN, Mavrakos SA (2016). Hydrodynamic analysis of an array of interacting free-floating oscillating water column (OWC's) devices. Ocean Engineering, 111:179-197.

18. Adnan, FAF; Hamylton, SM; Woodroffe, CD (2016). SurfSwash Interactions on a Low-Tide Terraced Beach, Journal of Coastal Research, SI75: 348-352.

19. Sheng WN, Alcorn R, Lewis A (2015). Optimising power take-offs for maximizing wave energy conversions. In: the 30th International Workshop on Water Waves and Floating Bodies, Bristol, UK.

20. Jakubowski, M (2015). Influence of pitting corrosion on fatigue and corrosion fatigue of ship and offshore structures, part II: load - PIT -crack interaction. Polish Maritime Research, 22(3): 57-66.

21. Sheng WN, Lewis A (2016). Power takeoff optimization for maximizing energy conversion of wave-activated bodies. IEEE Journal of Oceanic Engineering, 2016:1-12.

\section{CONTACT WITH AUTHOR}

Hengxu Liu

E-mail: liuhengxu@hrbeu.edu.cn 